\title{
ÉTICA DE LA INVESTIGACIÓN INTERNACIONAL: EL PROBLEMA DE LAJUSTICIA HACIA LOS PAÍSES MENOS DESARROLLADOS
}

\begin{abstract}
Resumen: En el presente, para proteger a las poblaciones de los países en desarrollo del peligro de explotación por patrocinadores de investigación internacional, se debe recurrir a los conceptos de "justicia distributiva" y "justicia como reciprocidad". El primero requiere que los riesgos y beneficios de las investigaciones sean repartidos con equidad (dar a cada uno lo que necesita) entre todas las personas o los grupos de la sociedad. El segundo demanda que los sujetos de investigación reciban beneficios por el hecho de participar. No se justificaría que si un sujeto paciente ha recibido placebo, al finalizar el estudio no reciba el medicamento cuyo efecto terapéutico ha sido probado por el estudio. Existe explotación cuando las personas o las agencias ricas o poderosas se aprovechan de la pobreza, debilidad o dependencia de otros, usándolos para alcanzar sus propias metas (las de los ricos o poderosos) sin beneficios adecuados para compensar a los individuos o los grupos que son dependientes o menos poderosos. Las Pautas Éticas Internacionales CIOMS y el Documento UNAIDS norman para que los productos de una investigación queden, razonablemente, disponibles en el país donde se realiza la investigación, y el documento UNAIDS especifica, incluso, que una vacuna para el SIDA se ponga al alcance de otras poblaciones que tengan un alto grado de infección con VIH, pero no existe una fuerza legal que garantice que esto se lleve a cabo. Sin embargo, se pueden utilizar los instrumentos de los derechos humanos para justificar la existencia de obligaciones de justicia como el artículo 12 del Tratado Internacional de los Derechos Económicos, Sociales y Culturales: "Derecho al más alto nivel de salud física y mental que pueda alcanzarse". Requiere que las naciones tomen pasos específicos, incluyendo prevención, tratamiento y control de varios tipos de enfermedades y, también, la creación de condiciones que asegurarían atención y servicios médicos para todos en caso de enfermedad.
\end{abstract}

Palabras Clave: Ética, investigación, justicia

\section{INTERNATIONAL RESEARCH ETHICS: THE PROBLEM OF THE JUSTICE TOWARD THE LESS DEVELOPED} COUNTRIES

\begin{abstract}
Today, in order to protect the population of developing countries from the risk of exploitation by international research sponsors, we must recur to the concepts of "distributive justice" and "justice as reciprocity". "Distributive justice" requires that the risks and benefits of research be distributed with equity (give to everyone what he/she needs) among all persons and groups in society. "Justice and reciprocity" requires that all research subjects receive benefits because of their participation. It will not be justified that if one subject patient has received placebo, at the end of the study he/she will not receive the drug that was therapeutically proved by the research. There is exploitation when rich or powerful persons or agencies take advantage of the poverty, weakness or dependency of others using them for their own goals (those of the rich or powerful) without adequate benefits to compensate the individuals or groups depending or less powerful. The International Ethical Guidelines CIOMS and the Document UNAIDS establish that the products of research must be made reasonably available in the country where the research takes place. UNAIDS document specifies that an AIDS vaccine must be made available to other populations with a high risk for VIH infection, but there is no legal empowerment to guarantee that this takes place. Nevertheless, human rights could be used as an instrument to justify the existence of justice obligations. This is the case of the International Treaty for Economical, Social and Cultural Rights article 12: "Right to the highest level of physical and mental health that could be reached". It requires that the nations take specific steps, including prevention, treatment and control of various types of diseases, and the creation of the conditions that assure medical care and services to all.
\end{abstract}

Key Words: Ethics, research, justice

\section{ÉTICA DA PESQUISA INTERNACIONAL: O PROBLEMA DA JUSTIÇA EM RELAÇ̃̃O AOS PAÍSES MENOS DESENVOLVIDOS}

Resumo: Atualmente para proteger as populações dos países em desenvolvimento do perigo da exploração por patrocinadores de pesquisa internacional, deve-se recorrer aos conceitos de "justiça distributiva" e "justiça como reciprocidade". O conceito de "justiça distributiva" exige que os riscos e benefícios das pesquisas sejam repartidos com equidade (dar a cada um o que necessita) entre todas as pessoas ou grupos da sociedade. O conceito de "justiça como reciprocidade" exige que os sujeitos da pesquisa recebam os benefícios pelo fato de participar. Não se justificaria que se um sujeito paciente tenha recebido placebo, ao finalizar o estudo não receba o medicamento cujo efeito terapêutico foi provado pelo estudo. Ocorre exploração quando as pessoas ou as agências ricas ou poderosas se aproveitam da pobreza, debilidade ou dependência dos outros, usando-os par alcançar suas próprias metas (as dos ricos ou poderosos), sem benefícios adequados para compensar aos indivíduos ou os grupos que são dependentes e menos poderosos. As diretrizes éticas internacionais CIOMS e o documento UNAIDS estabelecem normas com o objetivo de que os produtos de uma pesquisa fiquem razoavelmente disponíveis no país onde se realiza a pesquisa e o documento UNAIDS especifica, inclusiva, que uma vacina para a AIDS seja disponibilizada ao alcance de outras populações que tenham um alto grau de infeção com HIV, porém não existe uma força legal que garanta que isto se leva a cabo. Sem dúvida, pode-se justificar a existência de obrigações de justiça como o artigo 12 do tratado Internacional dos Direitos Econômicos, Sociais e Culturais: "Direito ao mais alto nível de saúde física e mental que se possa alcançar". Este tratado exige que as nações tomem passos específicos, incluindo prevenção, tratamento e controle de vários tipos de enfermidades e também, a criação de condições que assegurariam a atenção e serviços médicos para todos em caso de enfermidade. Palavras chave: Ética, pesquisa, justiça

* Department of Epidemiology and Social Medicine. Albert Einstein College of Medicine

Correspondencia: macklin@aecom.yu.edu 


\section{Introducción}

Hace muchos años ya existía preocupación sobre varios aspectos de las investigaciones con seres humanos. Estas preocupaciones eran generales y no específicamente en relación con la población de los países en desarrollo. Lo más común, sin duda, era el requisito para obtener consentimiento informado de los sujetos potenciales antes de iniciar una investigación. En el pasado la preocupación principal era la protección de la población en los países menos desarrollados con el fin de que no fuera explotada. La forma de explotación en este contexto consiste en experimentar con seres humanos sin su conocimiento y consentimiento. Aunque los comités de ética y los bioeticistas continúan atentos a esto, otras consideraciones han surgido recientemente en la categoría de justicia: el concepto denominado "justicia distributiva". El requerimiento básico de justicia distributiva exige que los riesgos y beneficios de las investigaciones sean distribuidos con equidad entre todas las personas o grupos de la sociedad. Por ejemplo, si los pobres son sometidos a los riesgos, y los ricos reciben los beneficios, se considera como violación de este principio.

Surge este problema cuando los investigadores provienen de un país industrial o el patrocinador es una empresa farmaceútica y la investigación se realiza en un país en desarro1lo. ¿Por qué se trata aquí de una cuestión de justicia? Porque en algunas investigaciones no se aplica el mismo nivel de cuidado a los pacientes que son sujetos de investigación en los países en desarrollo que aquél que se aplica en los países industriales. Daré algunos ejemplos más adelante.

Además, hay otra situación que no recibió atención hasta años recientes: lo que se debe a los sujetos de investigación cuando ésta concluye. Y una pregunta aún más problemática: ¿qué se le debe a la comunidad o al país en el que se realiza la investigación cuando ésta finaliza? En el pasado, cuando la investigación terminaba, los investigadores partían sin dejar beneficio alguno para los sujetos o para la población en general. Ahora esta situación es denominada "investigación de safari". Todos los beneficios que resultaban de las investigaciones que tenían éxito se dirigían a las poblaciones de los países ricos y ninguno a la gente donde se realizaba la investigación.

Hace cinco o seis años, algunas personas empezaron a prestar atención a ambas situaciones. Se realizó un gran debate con fuertes oponentes en ambos lados. Algunos, en estos momentos, consideran que el significado de "explotación" debe ser más amplio e incluir violaciones de justicia distributiva. Pero otros argumentan que la justicia no requiere que los niveles de cuidado sean los mismos dondequiera que se realicen las investigaciones. Además, esta última postura arguye que no existe ninguna obligación por parte de los patrocinadores de proveer beneficios (como medicinas) a la población del país donde se realiza la investigación. Este debate se encuentra en las revistas profesionales en medicina, bioética y en artículos escritos en la prensa popular. Vamos a examinar los argumentos de ambos lados en esta controversia.

\section{El nivel de cuidado: lo que se debe a los sujetos durante la investigación}

Hay varias preguntas en las que se plantea este tema. Cuando una investigación se realiza al mismo tiempo en un país industrial y en uno en desarrollo, ¿hay que proveer el mismo tratamiento a las personas en ambos países? Es decir, si un tratamiento no está disponible en un país pobre para los sujetos que quedan fuera de una investigación, pero el mismo tratamiento está disponible en un país industrial, ¿tienen los investigadores que proveerlo a los 
sujetos de la investigación en el país en desarrollo? No es aceptable, éticamente, no dar este tratamiento a los sujetos en el país en desarro1 o, porque existe una norma que dice: "No se permite exponer a los sujetos de investigación a una situación peor de aquélla en la que estarían si no se les involucrara en el estudio". Entonces, si los sujetos en el país industrial pueden obtener un tratamiento por su condición médica de quedar fuera de la investigación, ¿hay una obligación por parte de los investigadores (o del patrocinador) de proveerlo dentro de la investigación? Su condición médica no debe deteriorarse mientras sea posible tratarla.

Pero en la mayoría de los países pobres los sujetos de la investigación no tienen acceso al mismo tratamiento, puesto que no pueden pagar los medicamentos y el gobierno no provee este tratamiento en las clínicas públicas. Por lo tanto, la situación es diferente en los dos países. Si los sujetos en el país pobre no reciben el tratamiento no estarían peor que si no se involucraran en el estudio. Entonces, la norma mencionada no será violada. Debemos preguntar: ¿qué requiere la justicia?

Para empezar, tenemos que recordar que existen dos interpretaciones para los requerimientos de justicia. Una concepción de justicia es equidad y otra es igualdad. Estos conceptos no significan lo mismo. Podemos considerar, por ejemplo, dos sistemas posibles de seguro de salud. Una persona vive sola, es joven y su salud es excelente. Otra es vieja, tiene muchas enfermedades y tres hijos que también tienen enfermedades. El primer sistema de seguro de salud paga a todas las personas exactamente la misma cantidad de dinero cada mes, lo requieran o no, para gastos médicos. El segundo sistema paga a las personas que requieren dinero para gastos médicos, pero no paga a las personas que son saludables. El primer sistema utiliza un principio de justicia como igualdad: to- das las personas reciben la misma cantidad de dinero; el segundo, un principio de justicia como equidad: a cada persona según sus necesidades. ¿Cuál sistema es más justo? Es claro que un sistema de igualdad no siempre es más justo que un sistema de equidad. A veces una concepción es la apropiada para las circunstancias y, otras la concepción opuesta.

Regresemos ahora a las investigaciones multinacionales. En este debate, el lado que dice que los sujetos de los países en desarrollo deberían tratarse exactamente como los sujetos de los países industriales, aplica el principio de justicia como igualdad. Otro lado dice no: "La justicia no requiere que dos grupos se traten exactamente igual. Es suficiente que los grupos se traten con equidad". Además, argumentan, los sujetos de los países en desarrollo se tratan con igualdad con respecto a las otras personas de la sociedad: esto es, todas las que no son sujetos de las investigaciones.

Hay una complicación más. Existe un documento muy importante en relación con investigaciones en seres humanos: "La Declaración de Helsinki" contiene lineamientos para realizar los estudios. No es una ley y, por eso, no tiene la misma fuerza en todos los países del mundo. No obstante, es un documento muy respetado. La mayoría de los lineamientos no son controversiales, pero un párrafo ha causado el mismo debate que el asunto que estamos discutiendo ahora: "Los beneficios, los riesgos, las cargas y la efectividad de un método nuevo deberían probarse frente a los mejores métodos actuales, preventivos, diagnósticos y terapéuticos. Esto no excluye la utilización de placebo, o no tratamiento, en los estudios en los cuales no exista un método preventivo, diagnóstico y terapéutico comprobado".

¿Por qué este párrafo plantea un problema? Por las razones ya mencionadas: en los países 
en desarrollo la gente no tiene acceso a los mejores métodos cuando no se involucra en un estudio. Además, la afirmación sobre la utilización de placebo es muy problemática por varias razones. Una es que las empresas farmacéuticas quieren comparar su medicina nueva con un placebo. Creen que los datos de las investigaciones son más claros, la duración de los estudios más corta y la autoridad en los Estados Unidos -la FDA- prefiere que los estudios utilicen placebos cuando sea posible éticamente. Eso es lo central del asunto: ¿es ético o no utilizar placebo en lugar de una medicina probada cuando las investigaciones se realizan en los países en desarrollo? Los mismos estudios no podrían realizarse en los Estados Unidos, como hemos mencionado, porque los sujetos podrían tener acceso a los medicamentos probados fuera del estudio. En el gran debate un lado dice que si no es ético realizar un estudio en los Estados Unidos, no podría ser ético hacerlo con el mismo diseño en un país en desarrollo. Los oponentes señalan que es aceptable, éticamente, utilizar placebo cuando los sujetos no tendrían acceso a los métodos probados si no participaran en una investigación. El primer grupo responde que esto es "un doble estándar". El segundo está de acuerdo, pero agrega que, en esta situación, un doble estándar es aceptable éticamente.

¿Es necesario realizar investigaciones con seres humanos en los países en desarrollo? La respuesta es afirmativa, porque, primero, y lo más importante, existen enfermedades en los países tropicales que no se hallan en el Norte, por ejemplo, malaria, dengue, schistosomiasis y muchas otras. Si no se llevan a cabo investigaciones en las regiones en las que se presentan estas enfermedades no sería posible encontrar una cura o una vacuna preventiva. Segundo, aunque una enfermedad se encuentre en países en desarrollo e industriales -como el SIDA-, varias condiciones pueden ser diferentes. Por ejemplo, en cuanto al SIDA, hay diferencias en la ruta de transmisión del virus en África y en los Estados Unidos y, también, hay una diferencia en los tipos particulares (subtipos) del virus en estos lugares. Por esa razón, no es cierto que una vacuna que tenga éxito en los Estados Unidos lo tendrá también en los países de África. Además, puede haber diferencias en el estado de salud; por ejemplo, en algunos países la mayoría de la población sufre de desnutrición y, como resultado, los medicamentos arriesgan tener efectos diferentes.

Hay otra razón. El gobierno, es decir, el ministerio de salud, no acepta medicinas que no hayan sido probadas en su población; requieren que los estudios se realicen en su país. En este caso, ¿por qué no efectuar las investigaciones primero en los países industriales y, luego, en los países en desarrollo? Porque eso tiene como consecuencia una demora larga antes de que la población de un país en desarrollo obtuviera los beneficios de la investigación. Por lo tanto, muchas personas de países en desarrollo quieren que se ejecuten las investigaciones en sus países al mismo tiempo que en los Estados Unidos o en países de Europa.

Aunque existe un acuerdo amplio sobre la necesidad de realizar investigaciones con seres humanos en países en desarrollo, el desacuerdo continúa en cuanto al estándar de cuidado. Una causa - pero no la única- es la diferencia en las interpretaciones de lo que requiere justicia. Hay un principio formal de justicia que dice: "Trate los casos similares de manera similar, y trate los casos diferentes de manera diferente". El problema es que no queda siempre claro cuáles elementos deben ser estimados en una comparación. Vamos a considerar una investigación hipotética que sería realizada al mismo tiempo en los Estados 
Unidos y en Uganda. En los Estados Unidos habrá dos grupos: uno recibirá el medicamento experimental y el otro -el grupo de control- un medicamento probado para la enfermedad y aprobado por las autoridades. No se permite, éticamente, dar un placebo al grupo de control, porque existe un medicamento eficaz que los sujetos podrían recibir si no fueran involucrados en el estudio. En Uganda, sin embargo, aunque un grupo recibirá el medicamento experimental, el grupo de control recibirá un placebo. En este país, los sujetos no tendrían acceso al medicamento probado si no participaran en este estudio. En los Estados Unidos, el objetivo del estudio es averiguar si el medicamento experimental es tan eficaz, igual o mejor que el medicamento probado. En Uganda, el objetivo es determinar si el medicamento experimental es mejor que nada. ¿Son los dos casos similares o diferentes? Obviamente, son similares en algunos aspectos y diferentes en otros. El dilema es intentar determinar cuáles son los más relevantes cuando estamos tratando asuntos de justicia.

\section{Lo que requiere justicia después de finalizar una investigación}

Aunque algunos países en desarrollo tienen capacidad científica y ética e infraestructura para realizar investigaciones biomédicas, el hecho es que la mayoría de las que se llevan a cabo en países en desarrollo son patrocinadas por la industria farmacéutica. Además, los países industriales, frecuentemente, colaboran con investigadores de los países en desarrollo y también con la industria. Éste es el significado de las "investigaciones multinacionales".

Como ya he mencionado, surgen dos preguntas: ¿qué se le debe a los sujetos de investigación cuando concluye la investigación? Y una pregunta aún más problemática: ¿qué se le debe a la comunidad o al país en el que se realiza la investigación cuando finaliza? Primero vamos a examinar la pregunta más fácil.

El concepto de justicia relevante es el de "justicia como reciprocidad". Aquéllos que contribuyen a un proyecto merecen recibir algo a cambio. Los sujetos son sometidos a riesgos y dan su tiempo para participar en una investigación. Por supuesto, podrían recibir beneficios durante el estudio, pero también podrían no recibirlos. Por ejemplo, si un medicamento experimental no funciona, no hay beneficios para nadie. Además, si un estudio clínico tiene un grupo de control que recibe un placebo, los sujetos de ese grupo no gozan de beneficios. Si algunos necesitan un medicamento probado para su condición cuando finaliza una investigación, el concepto de justicia como reciprocidad requiere que lo reciban.

El párrafo 30 de la Declaración de Helsinki trata de este asunto. "Al final de la investigación, todos los pacientes que participan en el estudio deben tener la certeza de que contarán con los mejores métodos preventivos, diagnósticos y terapéuticos probados y existentes, identificados por el estudio". Esta afirmación no se encontraba en la versión previa de la Declaración de Helsinki, que se revisó en el año 2000. La inclusión de este párrafo muestra un reconocimiento de que "las investigaciones de safari” no son aceptables éticamente.

Vamos a considerar ahora la pregunta más problemática: ¿Cuál es la deuda hacia la comunidad o el país en el que se realiza la investigación cuando finaliza? Cuando finaliza con éxito en un país industrial, la mayoría de la población tendrá acceso al producto. O la gente puede pagar por sí misma o los seguros de salud o el sistema de salud pública lo hacen. Pero cuando culmina con éxito en un país en desarrollo, solamente una minoría tiene acceso al producto. Surge la pregunta: ¿deben los 
patrocinadores asegurar que la población tenga acceso a los beneficios de las investigaciones? Por lo menos, como ya hemos visto, los sujetos de investigación deben continuar recibiendo cualquier tratamiento que necesiten después de la investigación.

¿Qué debería pasar con las otras personas de la comunidad en la que se realizó la investigación o en el país entero? Este es el segundo gran debate. Un lado dice que no se le debe nada a la comunidad o a la población entera. Otro argumenta que el propósito principal de hacer la investigación es procurar beneficios a la población en el país en el que se realiza. Si no se ofrece beneficio alguno a la población, esto constituye explotación. Un vocero del primer lado responde: ¿cómo puede haber explotación si no se daña a nadie y los sujetos reciben los beneficios del tratamiento durante los estudios?

No podemos contestar las preguntas sobre explotación sin proveer una definición o, por lo menos, una idea aproximada del significado. ¿Cuáles son los criterios que definen “explotación"? Yo sugiero que consideremos lo siguiente: "Ocurre explotación cuando las personas -o las agencias ricas o poderosas-obtienen ventaja de la pobreza, debilidad o dependencia de los otros, usándolos para servir sus propias metas (las de las ricos o poderosos) sin beneficios adecuados para compensar a los individuos o a los grupos que son dependientes o menos poderosos". Entonces, en el contexto de las investigaciones con seres humanos, es posible que se dañe a los sujetos o sean tratados de una manera injusta y, al mismo tiempo, la comunidad en general en la que se realiza la investigación no sufra daños. No obstante, si se logra éxito en la investigación, alguien recibirá beneficios: el patrocinador, por lo menos, $\mathrm{y}$, probablemente, también los investigadores. Por lo tanto, surge la pregunta: ¿se trata de explotación cuando el poder entre dos grupos es desigual y el grupo más fuerte recibe beneficios mientras que el partido más débil recibe nada?

Vamos a regresar a la definición de "justicia distributiva": el requerimiento básico de la justicia distributiva establece que los riesgos y los beneficios de las investigaciones deben ser distribuidos con equidad entre todas las personas o los grupos de la sociedad. Por supuesto, los sujetos de las investigaciones son sometidos a riesgos. ¿Quién recibe los beneficios? Si la mayoría de la población de la comunidad o del país no tiene acceso a los productos de las investigaciones, pero la población en los países industriales sí lo tiene, se viola el principio de justicia distributiva.

Sin embargo, oponentes de esta conclusión dicen que, aunque los sujetos de la investigación contribuyeron en algo hacia el éxito del proyecto, la comunidad en general no lo hizo. Según este punto de vista, solamente aquéllos que contribuyen en algo merecen una compensación. Además, se dice que esta norma de justicia no existe en los Estados Unidos o, por lo menos, no es seguida. Nadie asegura que cuando una investigación finalice los medicamentos estarán disponibles para la comunidad o la población del país entero.

Eso es un hecho, pero no una justificación. Aunque la mayoría de la población de los Estados Unidos tiene acceso a los medicamentos que necesita, hay muchos pobres y diferentes grupos que carecen de seguros de salud. Por eso, la situación en ese país es tan injusta como en los países en desarrollo. La diferencia está en la cantidad de población sin poder adquisitivo ni seguro de salud.

¿Qué dicen los documentos internacionales con lineamientos éticos? Existen tres documentos principales que se aplican a las investigaciones con seres humanos: La Declaración de 
Helsinki; las Pautas Éticas Internacionales para Las Investigaciones Biomédicas que Involucran Seres Humanos, publicado por el Comité para las Organizaciones Internacionales de las Ciencias Médicas (CIOMS); y un documento más específico, Las Consideraciones Éticas en Las Investigaciones para una Vacuna Preventiva Contra VIH, publicado por UNAIDS (el Programa en VIH/SIDA de las Naciones Unidas). Este último es muy específico, porque trata de una sola enfermedad y de un método para prevenirla. Sin embargo, la mayoría de sus lineamientos pueden ser aplicados, asimismo, a otros tipos de investigación.

La Declaración de Helsinki no dice nada en cuanto a lo que se le debe a la comunidad o al país después de que una investigación concluye con éxito. Los dos otros documentos contienen mandatos casi enérgicos. Respecto de esto, CIOMS dice lo siguiente:

"Antes de realizar una investigación en una población o comunidad con recursos limitados, el patrocinador y el investigador deben hacer todos los esfuerzos para garantizar que:

- la investigación responde a las necesidades de salud y prioridades de la población o comunidad en que se realizará; y

- cualquier intervención o producto desarrollado, o conocimiento generado, estará disponible razonablemente para beneficio de aquella población o comunidad ${ }^{1 "}$.

Se han levantado críticas de varios tipos respecto de este lineamiento. Una dice que es demasiado débil; que los términos "disponible razonablemente" podrían ser interpretados de

Pauta 10: Investigación en poblaciones y comunidades con recursos limitados. Consejo de Organizaciones Internacionales de las Ciencias Médicas. Pautas éticas internacionales para la investigación biomédica en seres humanos. Santiago de Chile: Programa regional de Bioética OPS/OMS; 2003. tal manera, que el patrocinador o el investigador exprese: "El producto no puede hacerse disponible, porque es demasiado caro y, por eso, no es razonable proveerlo a la comunidad o a la población". Otra, dice que las palabras "disponible razonablemente" son demasiado vagas, porque no existe criterio alguno para determinar cuándo algo es razonable o no. Pero la crítica más fuerte ha sido planteada por los opositores a este lineamiento. ¿Qué argumentan ellos?

El punto principal plantea que tal requisito no se apoya en el pasado ni se ha hecho realidad en el presente. Para mí, este punto comete un error fundamental: confunde lo que existe o existió con lo que debe hacerse. Y la ética, precisamente, trata de lo que debe hacerse para mejorar las circunstancias de los seres humanos.

El tercer documento internacional afirma, aun con más fuerza, la obligación que existe al finalizar una investigación. El documento de UNAIDS dice: "Cualquier vacuna preventiva para VIH/SIDA que demuestre ser segura y eficaz debe ponerse a disposición, tan pronto como sea posible [...] a las otras poblaciones con alto riesgo de infección con VIH". Un comentario que sigue al lineamiento continúa diciendo: "La discusión sobre poner a disposición una vacuna debe empezar antes de que el estudio comience". El comentario especifica los elementos que deberían ser incluidos en esta discusión y, también, quiénes deberían ser sus miembros.

El problema con todos estos documentos que contienen lineamientos éticos que deben ser aplicados a las investigaciones con seres humanos es que no poseen fuerza de ley. No hay una organización internacional que pueda averiguar si los lineamientos están siendo obedecidos, ni una corte de justicia que pueda conducir los juicios. Por eso, es natural preguntar- 
se cuál es, exactamente, la importancia de estos documentos.

\section{Los derechos humanos y las investigaciones multinacionales}

Aunque muchas personas usan los términos "derechos humanos" de manera no precisa, en sentido estricto se refieren a los derechos que se encuentran en las declaraciones y, especialmente, en los tratados de la Organización de las Naciones Unidas (ONU). Cuando las naciones que son miembros de la ONU firman y ratifican los tratados, los gobiernos acuerdan seguirlos.

Ninguno de los tratados menciona "justicia" en forma explícita. Además, solamente un artículo en un tratado se refiere a las investigaciones con seres humanos. Artículo 7 del Pacto Internacional de los Derechos Civiles y Políticos: "Nadie será sometido a torturas ni a penas o tratos crueles, inhumanos o degradantes. En particular, nadie será sometido sin su libre consentimiento a experimentos médicos o científicos". La misma afirmación se halla en la Declaración de los Derechos Humanos, publicada en 1947, poco después de la segunda guerra mundial. Es claro que este artículo se refiere a los tipos de experimentos realizados por los nazis y no a la mayoría de las actuales investigaciones con seres humanos. No obstante, el punto principal es la necesidad de obtener consentimiento voluntario de los sujetos de las investigaciones. Este artículo no trata de justicia.

Pero, en otro documento, el Pacto Internacional de Derechos Económicos, Sociales, y Culturales, Artículo 12, se menciona un derecho que puede ser interpretado de alguna manera en conexión con la justicia:

"1.Los Estados Partes en el presente Pacto reconocen el derecho de toda persona al dis- frute del más alto nivel posible de salud física y mental.

2. Entre las medidas que deberán adoptar los Estados Partes en el Pacto, a fin de asegurar la plena efectividad de este derecho, figurarán las necesarias para:

a) La reducción de la mortalidad y de la mortalidad infantil, y el sano desarrollo de los niños;

b) El mejoramiento en todos sus aspectos de la higiene del trabajo y del medio ambiente;

c) La prevención y el tratamiento de las enfermedades epidémicas, endémicas, profesionales y de otra índole, y la lucha contre ellas;

d) La creación de condiciones que aseguren a todos asistencia médica y servicios médicos en caso de enfermedad".

Cuando alguna nación ratifica un tratado, se compromete a cumplir las obligaciones según sus requisitos. Esto es verdad en el caso de los países en desarrollo así como en el de los industrializados. El problema es que a las naciones pobres les faltan recursos para cumplir sus obligaciones. Sin embargo, este tratado de la ONU no requiere que las naciones cumplan sus obligaciones de manera inmediata. Al contrario, el documento permite "la realización progresiva" de los derechos que contiene. Además, hay cláusulas que imponen a las naciones más ricas ayudar a las naciones más pobres. Según una interpretación de las obligaciones estipuladas en este tratado internacional y en otros, se puede utilizar los instrumentos de los derechos humanos para justificar la existencia de las obligaciones de justicia. No obstante, ésta es un área nueva de estudio para los expertos en derechos huma- 
nos y para los bioeticistas. No será fácil poner este asunto en la agenda de las instancias que tienen la responsabilidad de revisar y juzgar el comportamiento de las naciones que han ratificado los tratados.

\section{Conclusión}

El problema de la justicia en las investigaciones multinacionales tiene muchos aspectos complejos. Aunque todo el mundo afirma su importancia, hay varias concepciones de jus- ticia y, también, interpretaciones diferentes de la misma. Al parecer, actualmente, está creciendo un consenso: los riesgos, inconveniencias y beneficios de las investigaciones con seres humanos deben ser compartidos por todos los grupos de una sociedad, así como por todas las naciones del mundo. Si no se realiza investigación con seres humanos, las personas no podrán ni curarse ni prevenir algunas enfermedades. Actualmente, las investigaciones son una actividad entre muchas en un mundo globalizado. 